\title{
Radial Forearm Wrapped-around Iliac Graft: Alternative Option for Thumb Reconstruction
}

\author{
A Shahrulazua, M Med Ortho (USM), WI Faisham, M Med Ortho (USM), \\ MZ Norazman, M Med Ortho (USM), W Zulmi*', MS Ortho (UKM) \\ Orthopaedics Department, Universiti Sains Malaysia Hospital, Kota Bharu, Malaysia \\ *Department of Orthopaedics and Traumatology, Universiti Kebangsaan Malaysia Medical Centre, \\ Kuala Lumpur, Malaysia
}

\begin{abstract}
We present a case in which a patient with well-differentiated squamous cell carcinoma of the dominant thumb underwent resection at the level of metacarpo-phalangeal joint and primary reconstructive surgery using a tricortical iliac bone graft with radial forearm wrapped-around pedicle flap. This single stage resection and reconstructive procedure was simple and relatively easy to perform, without the need for microsurgical expertise. Detailed surgical procedures and excellent functional result are described.
\end{abstract}

Key Words:

Squamous Cell Carcinoma, Thumb Resection, Thumb Reconstruction, Forearm Flap, Iliac Graft

\section{INTRODUCTION}

The incidence of squamous cell carcinoma in the thumb is rare. The prime objective of treatment is to control the lesion by wide resection of the tumour and to provide a functional reconstruction. In order to maintain normal thumb function which contributes to about $40 \%$ of hand function, the newly reconstructed thumb must possess: an adequate length for opposition; stability for resistance to other finger movement; and, sufficient abduction ability to allow functional opposition ${ }^{1}$. Mobility, particularly in the carpo-metacarpal joint, tactile sensibility and appearance of the thumb are desirable but are only secondary objectives ${ }^{1}$.

Several methods utilising non-microsurgical techniques or microvascular skills have been described for thumb reconstruction. These include phalangisation, distraction lengthening procedures, osteoplastic reconstruction, osseointegrated thumb prosthetic replacement, pollicisation, and composite free tissue transfer such as toe-to-thumb transfer, osteocutaneous radial forearm flap and first dorsal metacarpal artery island flap ${ }^{1,2}$. As each technique has certain advantages and disadvantages, the choice of surgery will depend on patient's age and gender, the level of amputation, presence and nature of injuries to other fingers, occupational and social factors, and the availability of tissues for reconstruction ${ }^{1}$. We describe here a variation of osteoplastic reconstructive surgery using an iliac bone graft with a radial forearm wrapped-around flap as a one-stage treatment procedure following resection of a cancerous thumb at the level of metacarpo-phalangeal joint.

\section{CASE REPORT}

A 67-year-old farmer sustained a chronic intermittently discharging wound on his dominant right thumb after being stung by a catfish 3 years previously. Over the years, the wound slowly grew into a painless fungating mass associated with itchiness. Examination revealed a $5 \mathrm{~cm}$ fungating mass over his right thumb with surrounding indurated skin. There was hardly any movement at the inter-phalangeal joint, but he was still able to freely move the metacarpo-phalangeal joint. Sensation was impaired distally from the level of proximal phalanx. There was no evidence of lymphadenopathy or any other distant metastasis.

Plain radiographs showed a complete destruction of the distal phalanx and part of the distal end of the proximal phalanx of the right thumb (Figure 1). No evidence of lung metastases was seen on chest radiograph or CT scan. A fourquadrant wedge biopsy confirmed a well-differentiated squamous cell carcinoma.

Excision of the tumour was achieved through amputation of the thumb by disarticulation at the metacarpo-phalangeal joint (Figure 2a). The distal surface of the first metacarpal was trimmed to create an oblique surface anteromedially, for abducted positioning of the graft. A tricortical bone graft was harvested from the iliac wing and sculpted to the measured length of the contralateral normal thumb. The graft, with its cancellous surface facing the palm, was then secured with an L-shape mini-plate to the metacarpal bone (Figure 2b). Abduction function was reinforced by tenodesing the abductor pollicis tendon to the graft. A distally-based radial forearm flap was raised from the volar aspect of the 


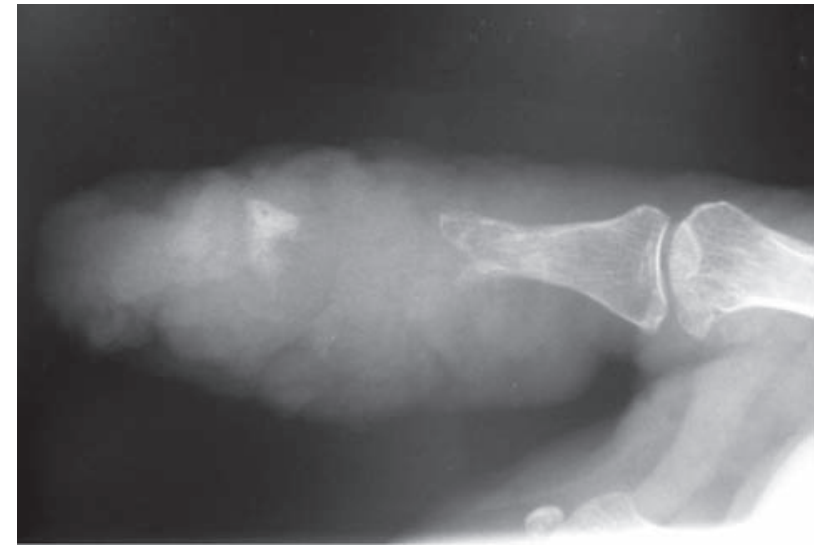

Fig. 1: Radiological appearance of the thumb showing a complete destruction of phalanges by the carcinoma.

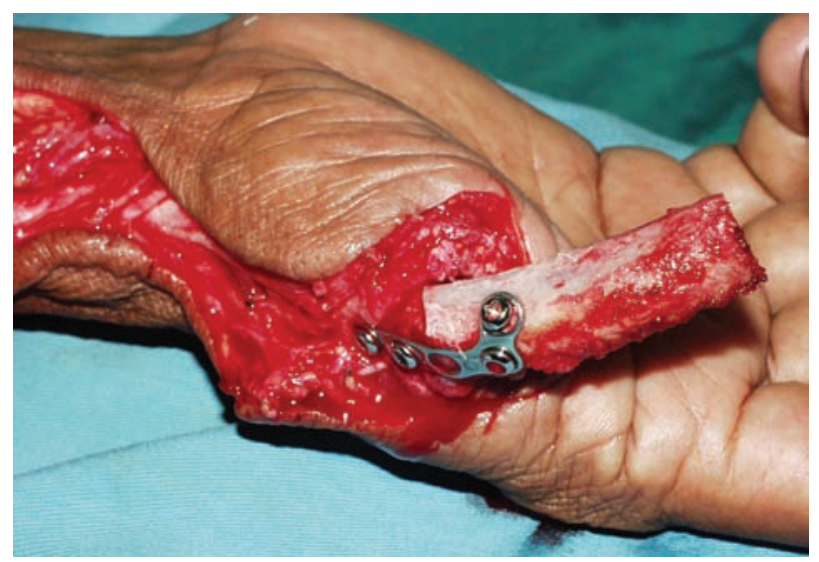

Fig. 2b: Intra-operative picture showing fixation of the iliac bone graft to the first metacarpal bone.

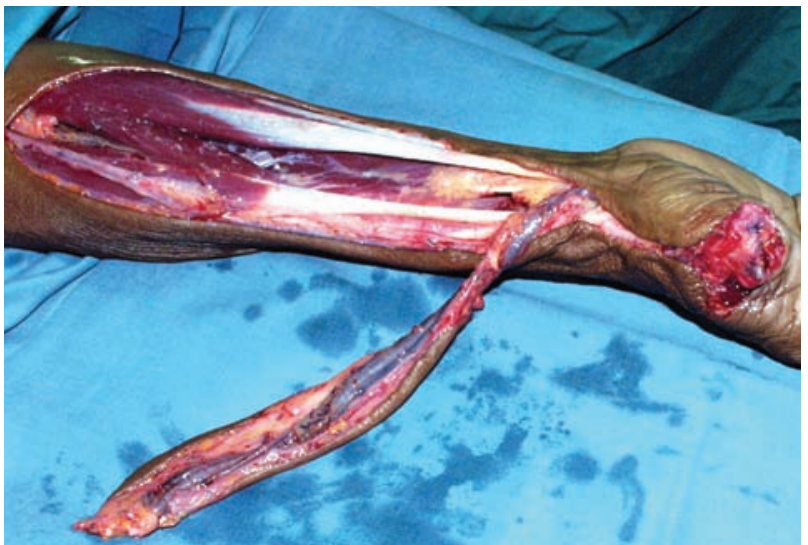

Fig. 2a: Intra-operative picture showing the amputated thumb and the raised radial forearm flap.

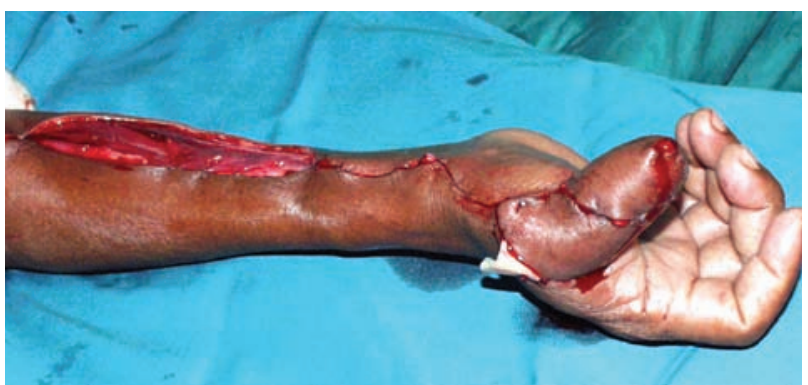

Fig. 2c: Intra-operative picture showing the final appearance of the radial forearm wrapped-around iliac graft flap.
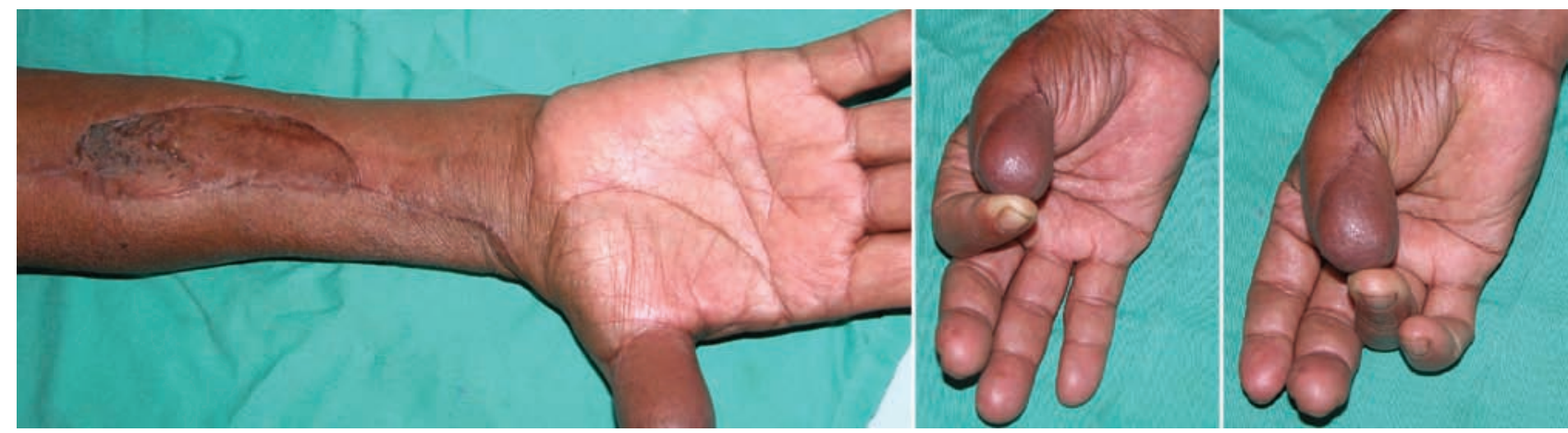

Fig. 3: Functional outcome at 3 months post-operative period. Patient demonstrated good range of motion and power, including pincer grip and oppositions.

ipsilateral forearm and wrapped-around the graft to provide soft tissue cover (Figure 2c). The donor site was closed with split-thickness skin graft. Prior to undertaking the radial forearm flap, an Allen test was conducted to confirm the patency of ulnar artery contribution to hand circulation.

Final histology of the resected thumb specimen confirmed a well-differentiated squamous cell carcinoma with no vascular or lymphatic invasion and a free surgical margin was achieved. Physiotherapy of the thumb was initiated on day one post-operatively, and continued until satisfactory recovery was achieved.

At 3 months post-surgery, the patient stated that he was very satisfied with the function of his new thumb. He had already returned to his previous work and was able to perform his normal activities of daily living. Mobility and strength of the thumb at the carpo-metacarpal joint consisting of flexion, extension, abduction, adduction and opposition were good (Figure 3). There were no signs of local recurrence or 
systemic spread, and radiographs of the thumb did not show any sign of bone graft resorption at one year following the surgery.

\section{DISCUSSION}

Squamous cell carcinoma is the most commonly seen hand malignancy, representing 58 to 90 percent of cases ${ }^{3}$. It commonly occurs in the 6th decade of life and is 4 times more common in men than in women ${ }^{3}$. The carcinoma rarely metastasises, and treatment is comprised of total excision to achieve tumour-free margins of at least $0.5 \mathrm{~cm}$ for a small lesion, and $3 \mathrm{~cm}$ for a fixed lesion ${ }^{3}$. Amputation is usually necessary for the latter. Reported recurrence rates vary from $7 \%$ to $22 \%{ }^{3}$. The association of squamous cell carcinoma as a complication of chronic non-healing ulcer has frequently been documented and this is further illustrated in our case report. Published literature reports on resection of squamous cell carcinoma of thumb and accompanying management choices are however scarce.

This case report highlights the potential for occurrence of squamous cell carcinoma in a thumb and describes a treatment option via resection along with a one-stage reconstructive procedure using a tricortical iliac bone graft and a radial forearm flap to wrap around the graft. This procedure is relatively easy to perform, without the need for lengthy and complicated microsurgical techniques. It is a useful single stage operation for those who refuse more complex thumb reconstruction procedures, which may include sacrifice of other digits or toes. Surgery is confined to a single limb, which remains free from any abnormal attachment to the body as seen when using a tube pedicle graft reconstructive method. The technique described here permits freedom of movement and better control of oedema, early mobilisation and rehabilitation. The immediate coverage of the bone graft with a well vascularised flap allows a permanent blood supply to be maintained, reducing the risk of bone graft resorption which can make the pulp unstable.

Another option for single stage thumb reconstruction which does not require microsurgical expertise uses a composite radial forearm flap ${ }^{2}$. However, this has the same advantages as the procedure described herein, but at the expense of a more considerable cosmetic defect at the donor site. There is also a high incidence of distal radius fracture associated with this composite radial forearm flap technique ${ }^{4}$, complicating the management and prolonging the time required until the patient could return to work.

Disadvantages of the present procedure are that there is no motion except at the basal joint, poor sensibility, bulky appearance of the thumb, and absence of a nail. On the other hand, the mere presence of a mobile carpo-metacarpal joint in a rigid reconstructed thumb still proves functionally useful ${ }^{1,2}$. Other disadvantages are those associated with the radial forearm donor site such as poor cosmetic end-result and the possible occurrence of hyperaesthetic area and/or neuromata formation. These potential problems can be minimised by using the volar aspect of the forearm, avoiding the radial border ${ }^{4}$. Radial forearm flap also requires sacrifice of a major artery of the hand and may result in a vascular steal syndrome and acute ischaemia of the hand. Nevertheless, studies using digital temperature, Doppler flow and colour duplex imaging have demonstrated that the loss of the radial artery does not cause any significant impairment to the hand circulation ${ }^{5}$.

The aim of treatment in the present patient was to resect the tumour and reconstruct a functional thumb to allow him to return to his work and earn his living again. Thus, appearance was of secondary importance. Thumb sensibility for fine manipulation, protection and assessment of objects was not crucial as the opposing fingers were/are still sensate $^{1}$. Even with the absence of the distal joint and poor thumb sensibility, the patient could still achieve good function and strength with his new thumb, sufficient for him to regain his previous employment.

In conclusion, resection and reconstruction procedure as described offers a quick, relatively inexpensive, and simple one-stage procedure, devoid of any complicated microsurgical techniques, whilst not compromising the essentials of length, stability and opposability needed in a newly reconstructed thumb for a manual labourer. 


\section{REFERENCES}

1. Lister G. The choice of procedure following thumb amputation. Clin Orthop Rel Res 1985; 195: 45-51.

2. Kleinman WB, Strickland JW. Thumb Reconstruction. In: Green DP, Hotchkiss RN, Pederson WC, eds. Green's operative hand surgery. Vol II, 4th ed. Philadelphia: Churchill Livingston. 1999: 2069-170.

3. Calandruccio JH, Jobe MT. Tumors and tumorous conditions of hand. In: Canale ST, ed. Campbell's operative orthopaedics. Vol IV, 10th ed. St. Louis: Mosby. 2003: 3791.

4. Bardsley AF, Soutar DS, Elliot D, Batchelor AG. Reducing morbidity in the radial forearm flap donor site. Plast Reconstr Surg 1990; 86: 287-92.

5. Ciria-Llorens G, Gomez-Cia T. Hand blood supply in radial forearm flap donor extremities: a qualitative analysis using Doppler examination. J Hand Surg [Br] 2001; 26: 125-8. 